\title{
Tratamiento farmacomecánico dirigido por catéter de la trombosis venosa profunda: informe de casos
}

\section{Pharmacomechanical catheter-directed management of deep venous thrombosis: case series}

\author{
Juan D. Granobles-Molina', Jorge F. Tobar², Juan C. Chaves³ y Ángela Ma. Merchán-Galvis ${ }^{4 *}$ \\ ${ }^{1}$ Departamento de Ciencias Quirúrgicas; ${ }^{2}$ Departamento de Ciencias Quirúrgicas, Unidad Vascular, Clínica La Estancia; ${ }^{3}$ Programa de Medicina; \\ ${ }^{4}$ Departamento de Medicina Social y Salud Familiar. Universidad del Cauca, Popayán, Colombia
}

\begin{abstract}
Resumen
Objetivo: Describir los desenlaces en pacientes con trombosis venosa profunda de miembros inferiores a quienes se les realizó trombectomía o trombólisis con y sin angioplastia con y sin filtro de vena cava inferior. Resultados: Se incluyeron a 28 pacientes, en su mayoría mujeres (71.4\%), con edad promedio de 46.7 años, que recibieron tratamiento con trombólisis + trombectomía mecánica, que a su vez se relacionó con mayor permanencia en cuidados intensivos $(p=0.023)$. Los principales factores de riesgo fueron diabetes mellitus/HTA, TVP previa y operación ortopédica. El segmento venoso comprometido con mayor frecuencia fue el iliofemoral, que se vinculó con antecedente quirúrgico $(p=0.027)$, compresión venosa ilíaca $(p=0.044)$ y uso de filtro de vena cava inferior $(p=0.044)$, pero no con complicaciones $(p=0.089)$. Hasta $30 \%$ de los pacientes presentó retrombosis seis meses después del primer procedimiento; $7 \%$ de los pacientes tuvo síndrome postrombótico grave. Conclusiones: Este registro sugiere que el tratawmiento farmacomecánico dirigido por catéter es seguro y efectivo en la TVP y reduce los tiempos de estancia hospitalaria, aparición y gravedad del síndrome postrombótico.
\end{abstract}

Palabras clave: Trombosis venosa. Fibrinólisis. Trombólisis mecánica. Cateterismo venoso periférico. Síndrome postrombótico. Síndrome de May-Thurner.

\begin{abstract}
Objective: To describe the outcomes in patients with deep vein thrombosis of the lower limbs who underwent thrombectomy or thrombolysis with and without angioplasty with and without an inferior vena cava filter. Results: Twenty-eight patients were included, mostly women $(71.4 \%)$, with an average age of 46.7 years, who received treatment with thrombolysis + mechanical thrombectomy, which in turn was associated with a longer stay in intensive care $(p=0.023)$. The main risk factors were diabetes mellitus / HT, previous DVT and orthopedic operation. The most frequently involved venous segment was the iliofemoral segment, which was associated with a surgical history $(p=0.027)$, iliac venous compression $(p=0.044)$ and use of an inferior vena cava filter $(p=0.044)$, but not with complications $(p=0.089)$. Up to $30 \%$ of the patients presented rethrombosis six months after the first procedure; $7 \%$ of the patients had severe post-thrombotic syndrome. Conclusions: This registry suggests
\end{abstract}

\section{Correspondencia:}

*Ángela Ma. Merchán-Galvis

E-mail: angelamerchan@ unicauca.edu.co

0377-4740/@ 2020 Sociedad Mexicana de Angiología y Cirugía Vascular y Endovascular, A.C. Publ bajo la licencia CC BY-NC-ND license (http://creativecommons.org/licenses/by-nc-nd/4.0/).
Available online: 27-01-2021 Rev Mex Angiol. 2020;48(4):107-116 www.RMAngiologia.com

\footnotetext{
(1)
} 
Rev Mex Angiol. 2020;48(4)

that catheter-directed pharmacomechanical treatment is safe and effective in DVT and reduces hospital stay times, onset and severity of post-thrombotic syndrome.

Key words: Venous thrombosis. Fibrinolysis. Mechanical thrombolysis. Catheterization peripheral. Postthrombotic syndrome. May-Thurner Syndrome.

\section{Introducción}

El tromboembolismo venoso (TEV) se refiere a un coágulo anormalmente producido en el sistema venoso e incluye tromboembolismo pulmonar (TEP) y trombosis venosa profunda (TVP) 1 . Afecta al $0.1 \%$ a $0.2 \%$ de la población general por año ${ }^{1}$, con una incidencia de 117 casos por cada 100,000 habitantes/año en Estados Unidos y 63 casos por cada 100,000 habitantes/ año en Europa ${ }^{2}$. En Colombia ya producía en 1990 más de 100,000 hospitalizaciones/año ${ }^{3}$. Su aparición se debe a factores que inducen hipercoagulabilidad, estasis o daño de la pared vascular $r^{4,5}$. La TVP iliofemoral no tratada representa un riesgo cercano al 50\% de desarrollar TEP sintomático en los siguientes tres meses $^{6}$ y puede llevar al síndrome postrombótico (SPT) que puede ser grave en el $5 \%$ al $10 \%$ de los casos?.

El tratamiento habitual ha sido la anticoagulación sumada a medias elastocompresivas y deambulación temprana; sin embargo, son medidas que no modifican directamente al trombo y la carga generada sobre la vena ${ }^{6}$. En la década de 1980 surgió como opción terapéutica la trombólisis sistémica, que mejoró la eliminación de coágulos y redujo la extensión de la trombosis, pero con mayor riesgo de sangrados mayores ${ }^{8}$. Han aparecido nuevas técnicas para inyectar el trombolítico directamente en el trombo a través de un catéter, lo que reduce la dosis del fármaco y por ende el riesgo de sangrado. Están disponibles también nuevos dispositivos que emplean la trombectomía mecánica con catéter ${ }^{6}$, lo que facilita el uso de la angioplastia cuando persisten obstrucciones venosas que pueden predisponer a TVP recurrente $^{9}$. Las guías internacionales ${ }^{10,11}$ y el consenso colombiano de fibrinólisis selectiva con catéter en la enfermedad vascular tromboembólica ${ }^{9}$ recomiendan la trombólisis localizada farmacológica 0 mecánica en pacientes con TVP iliofemoral; por lo tanto, el propósito fue describir los desenlaces de los pacientes con TVP de miembros inferiores tratados con técnicas endovasculares guiadas por catéter con o sin angioplastia, en una clínica de tercer nivel de la ciudad de Popayán.

\section{Material y métodos}

Estudio descriptivo y ambispectivo realizado bajo parámetros de buena práctica clínica, ajustado a las normas éticas de la Declaración de Helsinki de 1975 y previa aprobación del comité de bioética de la Clínica La Estancia donde se realizó el estudio. La información se obtuvo de la revisión de historias clínicas y descripciones quirúrgicas de pacientes atendidos entre abril de 2015 y marzo de 2019. Los pacientes aceptaron participar del estudio mediante la firma del consentimiento informado.

Se incluyó a todos los pacientes mayores de 18 años que tenían TVP de miembros inferiores primera o recurrente, diagnosticada mediante ecografía Doppler o flebografía, con indicación de algún procedimiento endovascular terapéutico para TVP. Se excluyó a los pacientes con registros incompletos y aquéllos con TVP crónica relacionada con SPT. La información recolectada incluyó datos demográficos de los pacientes, antecedentes médicos y quirúrgicos, estancia hospitalaria, días de duración de los síntomas relacionados con TVP, segmento venoso comprometido por ecografía Doppler o flebografía, técnica endovascular usada, uso de angioplastia, estancia en unidad de cuidado intensivo (UCl), complicaciones relacionadas con el procedimiento, y forma de diagnóstico y tratamiento. También se incluyó información acerca de la recurrencia, reintervención y técnica endovascular empleada, número de controles ambulatorios, y tiempo de seguimiento luego de la primera intervención. Se estableció contacto telefónico con los pacientes para realizar la escala de Villalta y definir la presencia y gravedad de SPT.

En el procedimiento endovascular se efectuaba punción guiada por ecografía en la vena poplítea o safena mayor según fuera la posibilidad de acceso venoso en la cara medial del muslo distal. Se aplicó alteplasa a una dosis de carga de $0.01 \mathrm{mg} / \mathrm{kg}$ y dosis de mantenimiento de 0.05 a $1 \mathrm{mg} / \mathrm{h}$ en infusión continua por un máximo de $48 \mathrm{~h}$. Entre los dispositivos usados se hallaban balones semidistensibles o no distensibles Mustang (Boston) o Atlas (Bard Medical); para la aspiración se utilizaron dispositivos Indigo Cat 8 (Penumbra), 
Tabla 1. Antecedentes de los pacientes intervenidos con TDC/TFM diagnosticados de TVP

\begin{tabular}{|c|c|c|c|c|c|c|c|}
\hline & & \multicolumn{2}{|c|}{ Masculino ( $(n=8)$} & \multicolumn{2}{|c|}{ Femenino $(n=20)$} & \multicolumn{2}{|c|}{ Total $(n=28)$} \\
\hline & & n & $\%$ & $\mathbf{n}$ & $\%$ & n & $\%$ \\
\hline \multirow[t]{2}{*}{ Edad } & Media & 44.3 & & 47.7 & & 46.7 & \\
\hline & DS & 27.6 & & 14.8 & & 18.8 & \\
\hline \multirow[t]{8}{*}{ Antecedentes médicos } & DM/HTA & 0 & 0.0 & 5 & 25. & 5 & 17.9 \\
\hline & Anticonceptivos & 0 & 0.0 & 3 & 15.0 & 3 & 10.7 \\
\hline & TVP & 1 & 12.5 & 3 & 15.0 & 4 & 14.3 \\
\hline & TEP & 0 & 0.0 & 92 & 10.0 & 2 & 7.1 \\
\hline & Gestación/puerperio & 0 & 0.0 & 2 & 10.0 & 2 & 7.1 \\
\hline & Insuficiencia venosa & 2 & 25.0 & 0 & 0.0 & 2 & 7.1 \\
\hline & Insuficiencia renal & 1 & 12.5 & 0 & 0.0 & 1 & 3.6 \\
\hline & Ninguno & 4 & 50.0 & 5 & 25.0 & 9 & 32.1 \\
\hline \multirow[t]{7}{*}{ Antecedentes quirúrgicos } & Ortopedia & 2 & 25.0 & 2 & 10.0 & 4 & 14.3 \\
\hline & Cesárea & 0 & 0.0 & 4 & 20.0 & 4 & 14.3 \\
\hline & Histerectomía & 0 & 0.0 & 2 & 10.0 & 2 & 7.1 \\
\hline & Colecistectomía & 0 & 0.0 & 1 & 5.0 & 1 & 3.6 \\
\hline & Eventrorrafia & 0 & 0.0 & 2 & 10.0 & 2 & 7.1 \\
\hline & Varicectomía & 0 & 0.0 & 1 & 5.0 & 1 & 3.6 \\
\hline & Ninguno & 6 & 75.0 & 8 & 40.0 & 14 & 50.0 \\
\hline \multirow[t]{2}{*}{ Antecedentes neoplásicos } & Útero/cérvix & 0 & 0.0 & 2 & 10.0 & 2 & 7.1 \\
\hline & Ninguno & 8 & 100.0 & 18 & 90.0 & 26 & 92.9 \\
\hline
\end{tabular}

Neuron Max 088 (Penumbra) o Angiojet (Boston), así como el Stent Zilver Vein IQ (Cook) y Wallstent (Boston).

El éxito técnico se definió por la evidencia flebográfica de remoción de más del $50 \%$ del coágulo con la restauración del flujo venoso iliofemoral, preservación anatómica o funcional de la extremidad, supervivencia y mejoría de los síntomas, mientras que las complicaciones fueron la mortalidad, el sangrado mayor, sangrado menor y TEP. El sangrado mayor se definió como sangrado letal o intracraneal, intraespinal, intraocular, retroperitoneal, pericárdico, articular o intramuscular con síndrome compartimental, o bien caída del valor de la hemoglobina de al menos $2 \mathrm{~g} / \mathrm{dl}$ o que condujera a transfusión de 2 o más unidades de sangre completa 0 eritrocitos, en las 24 a $48 \mathrm{~h}$ tras el sangrado o sangrado del sitio quirúrgico que produjera una segunda intervención quirúrgica ${ }^{12}$. El sangrado menor fue cualquier hemorragia que no cumpliera los criterios anteriores ${ }^{13}$.
Para el análisis estadístico, las variables cualitativas nominales y ordinales se presentan como proporciones y para las variables cuantitativas, según fuera su distribución, se aplicaron medidas de tendencia central y dispersión. Para comparar las variables entre hombres y mujeres, así como analizar los factores vinculados con la presentación de la TVP, se usó la prueba estadística $\chi^{2}$ y se estableció el grado de significancia en un valor de $p \leq 0.05$. El paquete estadístico utilizado para el análisis de la información fue SPSS V.25.

\section{Resultados}

Se incluyó a 28 pacientes, todos con diagnóstico de TVP de miembros inferiores por ecografía Doppler 0 flebografía. Las mujeres constituyeron el 71.4\% $(n=20)$ y la edad promedio fue de 46.7 años. Los principales factores de riesgo se describen en la tabla 1. De los antecedentes médicos, el más común en toda la 
Tabla 2. Hallazgos clínicos de los pacientes intervenidos con TDC diagnosticados de TVP

\begin{tabular}{|c|c|c|c|c|c|c|c|}
\hline & & \multicolumn{2}{|c|}{ Masculino } & \multicolumn{2}{|c|}{ Femenino } & \multicolumn{2}{|c|}{ Total } \\
\hline & & n & $\%$ & n & $\%$ & n & $\%$ \\
\hline \multirow[t]{2}{*}{ Días de síntomas } & Media & 10.9 & & 15.4 & & 14.1 & \\
\hline & DS & 6.6 & & 10.3 & & 9.5 & \\
\hline \multirow[t]{3}{*}{ Días de síntomas agrupados } & $0-13$ & 6 & 75.0 & 9 & 45.0 & 15 & 53.6 \\
\hline & $14-20$ & 1 & 12.5 & 7 & 35.0 & 8 & 28.6 \\
\hline & 21 o más & 1 & 12.5 & 4 & 20.0 & 5 & 17.9 \\
\hline \multirow[t]{4}{*}{ Segmento afectado en Doppler } & Iliocavo & 1 & 12.5 & 1 & 5.0 & 2 & 7.1 \\
\hline & Iliofemoral & 6 & 75.0 & 18 & 90.0 & 24 & 85.7 \\
\hline & Femoropoplíteo & 0 & 0.0 & 1 & 5.0 & 1 & 3.6 \\
\hline & Ninguno & 1 & 12.5 & 0 & 0.0 & 1 & 3.6 \\
\hline \multirow[t]{2}{*}{ Segmento afectado en flebografía } & Iliocavo & 1 & 12.5 & 4 & 20.0 & 5 & 17.9 \\
\hline & Iliofemoral & 7 & 87.5 & 16 & 80.0 & 23 & 82.1 \\
\hline \multirow[t]{2}{*}{ Compresión de vena ilíaca } & Sx de May-Thurner & 2 & 25.0 & 5 & 25.0 & 7 & 25.0 \\
\hline & Ninguna & 6 & 75.0 & 15 & 75.0 & 21 & 75.0 \\
\hline
\end{tabular}

población fue la diabetes mellitus o la hipertensión arterial, con $17.9 \%(n=5)$, seguido por el antecedente de TVP con un $14.3 \%(n=4)$; en el $50.0 \%$ de los pacientes masculinos y en $25.0 \%$ de las mujeres $(n=5)$ no se logró identificar un antecedente médico relacionado con TVP ( $p=0.062)$. El antecedente quirúrgico más común fue la operación ortopédica con 14.3\% $(n=4)$, más frecuente en hombres que en mujeres (25.0\% vs $10.0 \%$, respectivamente). El $20.0 \%(n=4)$ de las mujeres tenía cesárea reciente. No se encontraron diferencias estadísticamente significativas entre los sexos. Se identificó cáncer de endometrio o cuello uterino en casi $10 \%$ de las mujeres $(n=2)$ (tabla 1 ).

Los síntomas duraron en promedio 14.1 días antes de la primera intervención. Un $53.6 \%$ de los pacientes $(n=15)$ tuvo cuadros de TVP aguda (síntomas de menos de 14 días); $28.6 \%$ tuvo TVP subaguda $(n=8)(14$ a 20 días de duración) (tabla 2). No existió diferencia significativa entre los sexos. En el Doppler, el $85.7 \%$ de los pacientes $(n=24)$ mostraba un compromiso venoso iliofemoral, mayor en mujeres ( $90.0 \%$ vs $75.0 \%)$. En la flebografía, el segmento comprometido con más frecuencia fue el iliofemoral con $82.1 \%$ de los casos $(n=23)$, sin diferencias significativas con el Doppler $(p=0.082)$. El síndrome de May-Thurner se presentó en $25.0 \%$ de la población $(n=7)$, sin diferencia entre sexos (tabla 2).
En $46.4 \%$ de los pacientes se empleó la combinación de trombólisis + tromboaspiración $(n=13)$, con mayor uso en hombres respecto de las mujeres $(62.5 \%$ vs $40.0 \% ; n=5$ y $n=8$, respectivamente). Se insertó filtro de vena cava inferior en $40.0 \%$ de las mujeres $(n=11)$ y $37.5 \%$ de los hombres $(n=3)$. Se practicó angioplastia con stent en $78.6 \%$ de los pacientes $(n=22)$ (tabla 3). El $32.1 \%$ de los pacientes $(n=9)$ tuvo complicaciones relacionadas con el procedimiento. Las más comunes fueron hematoma en el sitio de punción, en $10.7 \%$ de los pacientes $(n=3)$, seguido por la caída de la hemoglobina en $7.1 \%(n=2)$, sin diferencias significativas entre géneros $(p=0.051)$. El diagnóstico se estableció la mayoría de las veces mediante ecografía $(44.4 \%, n=4) 0$ análisis de laboratorio $(33.3 \%, n=3)$. Un hombre, que corresponde a $3.6 \%$ de los pacientes, falleció como complicación por la TVP en relación con un acceso femoral de hemodiálisis y la intervención realizada (tabla 3). Los pacientes se hospitalizaron en promedio 15.1 días, con una media en UCI de 2.8 días, sin diferencia por sexo. El principal síntoma al momento del egreso fue edema (tabla 4). Un $28.6 \%$ de los pacientes $(n=7)$ sufrió recurrencia, en ningún caso dentro de las primeras $48 \mathrm{~h}$ luego del primer procedimiento, sin diferencia entre sexos $(p=0.053)$. El $75.0 \%$ de los hombres $(n=3)$ y el $100 \%$ de las mujeres $(n=4)$ que tuvieron retrombosis se diagnosticaron 
Tabla 3. Descripción del procedimiento realizado a pacientes diagnosticados de TVP

\begin{tabular}{|c|c|c|c|c|c|c|c|}
\hline & & \multicolumn{2}{|c|}{ Masculino } & \multicolumn{2}{|c|}{ Femenino } & \multicolumn{2}{|c|}{ Total } \\
\hline & & n & $\%$ & n & $\%$ & n & $\%$ \\
\hline \multirow[t]{3}{*}{ Técnica endovascular } & Tromboaspiración & 2 & 25.0 & 7 & 35.0 & 9 & 32.1 \\
\hline & Trombólisis & 1 & 12.5 & 5 & 25.0 & 6 & 21.4 \\
\hline & Trombólisis + aspiración & 5 & 62.5 & 8 & 40. & 13 & 46.4 \\
\hline \multirow[t]{2}{*}{ Filtro vena cava inferior } & Sí & 3 & 37.5 & 8 & 40.0 & 11 & 39.3 \\
\hline & No & 5 & 62.5 & 12 & 60.0 & 17 & 60.7 \\
\hline \multirow[t]{2}{*}{ Angioplastia stent } & Sí & 7 & 87.5 & 15 & 75.0 & 22 & 78.6 \\
\hline & No & 1 & 12.5 & 5 & 25.0 & 6 & 21.4 \\
\hline \multirow[t]{7}{*}{ Complicaciones } & Ninguna & 4 & 50.0 & 15 & 75.0 & 19 & 67.9 \\
\hline & Descenso de la hemoglobina & 0 & 0.0 & 2 & 10.0 & 2 & 7.1 \\
\hline & Hematoma en sitio $\mathrm{QX}$ & 3 & 37.5 & 0 & 0.0 & 3 & 10.7 \\
\hline & Hematoma retroperitoneal & 0 & 0.0 & 1 & 5.0 & 1 & 3.6 \\
\hline & Muerte & 1 & 12.5 & 0 & 0.0 & 1 & 3.6 \\
\hline & TEP & 0 & 0.0 & 1 & 5.0 & 1 & 3.6 \\
\hline & Hematuria & 0 & 0.0 & 1 & 5.0 & 1 & 3.6 \\
\hline \multirow[t]{4}{*}{ Diagnóstico de la complicación } & Ecografía & 3 & 75.0 & 1 & 20.0 & 4 & 44.4 \\
\hline & Angio-TAC & 0 & 0.0 & 1 & 20.0 & 1 & 11.1 \\
\hline & Laboratorio & 0 & 0.0 & 3 & 60.0 & 3 & 33.3 \\
\hline & Clínica & 1 & 25.0 & 0 & 0.0 & 1 & 11.1 \\
\hline
\end{tabular}

Tabla 4. Días de hospitalización y síntomas al egreso de los pacientes intervenidos con TDC diagnosticados de TVP

\begin{tabular}{|c|c|c|c|c|c|c|c|}
\hline & & \multicolumn{2}{|c|}{ Masculino } & \multicolumn{2}{|c|}{ Femenino } & \multicolumn{2}{|c|}{ Total } \\
\hline & & $\mathbf{n}$ & $\%$ & $\mathbf{n}$ & $\%$ & n & $\%$ \\
\hline \multirow[t]{2}{*}{ Estancia hospitalaria } & Media & 14.6 & & 15.2 & & 15.1 & \\
\hline & DS & 5.1 & & 13.8 & & 11.9 & \\
\hline \multirow[t]{2}{*}{ Estancia UCI } & Media & 3.0 & & 2.7 & & 2.8 & \\
\hline & DS & 1.7 & & 2.2 & & 2.1 & \\
\hline \multirow[t]{2}{*}{ Estancia UCIN } & Media & 0.5 & & 1.6 & & 1.3 & \\
\hline & DS & 0.7 & & 2.5 & & 2.2 & \\
\hline \multirow[t]{4}{*}{ Síntomas al egreso } & Dolor & 0 & 0.0 & 2 & 10.0 & 2 & 7.1 \\
\hline & Edema & 6 & 75.0 & 10 & 50.0 & 16 & 57.1 \\
\hline & Fallecido & 1 & 12.5 & 0 & 0.0 & 1 & 3.6 \\
\hline & Asintomático & 1 & 12.5 & 8 & 40.0 & 9 & 32.2 \\
\hline
\end{tabular}

luego de 30 días (tabla 5). El 42.9\% de los pacientes tromboaspiración, otro trombólisis y el restante fue obcon recurrencia se llevó a un nuevo procedimiento en- jeto de angioplastia $(p=0.047)$. Una proporción de dovascular $(n=3)$, uno recibió trombólisis $+66.7 \%$ de los pacientes $(n=2)$ con retrombosis se 
Tabla 5. Resumen de los segmentos venosos comprometidos y las intervenciones endovasculares llevadas a cabo en los pacientes con recurrencia de TVP

\begin{tabular}{|c|c|c|c|c|c|c|c|}
\hline & & \multicolumn{2}{|c|}{ Masculino } & \multicolumn{2}{|c|}{ Femenino } & \multicolumn{2}{|c|}{ Total } \\
\hline & & $\mathbf{n}$ & $\%$ & $\mathbf{n}$ & $\%$ & $\mathbf{n}$ & $\%$ \\
\hline \multirow[t]{2}{*}{ Retrombosis } & Sí & 4 & 50.0 & 3 & 15.0 & 7 & 25.0 \\
\hline & No & 4 & 50.0 & 17 & 85.0 & 21 & 75.0 \\
\hline \multirow{2}{*}{$\begin{array}{l}\text { Pacientes llevados a angioplastia con o sin stent durante } \\
\text { la reintervención }\end{array}$} & Sí & 2 & 66.7 & 0 & 0.0 & 2 & 66.7 \\
\hline & Angioplastia fallida & 1 & 33.3 & 0 & 0.0 & 1 & 33.3 \\
\hline \multirow[t]{3}{*}{ Técnica endovascular usada en la reintervención } & Trombólisis & 1 & 33.3 & 0 & 0.0 & 1 & 33.3 \\
\hline & Trombólisis + aspiración & 1 & 33.3 & 0 & 0.0 & 1 & 33.3 \\
\hline & Ninguna & 1 & 33.3 & 1 & 100.0 & 1 & 33.3 \\
\hline
\end{tabular}

sometió a angioplastia con o sin colocación de stent (tabla 5).

El tiempo de seguimiento fue en promedio de 8.2 meses en mujeres y 3.5 meses en hombres. Se realizó un seguimiento ambulatorio en $75.0 \%$ de los pacientes, $35.8 \%$ con tiempos de seguimiento de 3 a 12 meses $(n=10)$. En el período de estudio, 3 pacientes fallecieron luego del egreso (tabla 6). Al analizar los factores relacionados con la TVP, la elección del procedimiento y los resultados del tratamiento se encontró un nexo entre la afectación iliofemoral y el antecedente quirúrgico $(p=0.027)$, además de relación entre la compresión venosa iliaca y la afectación de este segmento en la flebografía ( $p=0.044$ ) y el uso de filtro de vena cava $(p=0.044)$, pero no con las complicaciones $(p=0.089)$. La trombólisis + aspiración se acompañó de mayor estancia en $\mathrm{UCl}(p=0.023)$.

\section{Discusión}

Se describió esta serie de casos con 28 pacientes diagnosticados con TVP de miembros inferiores, la mayoría mujeres y con edad aproximada de 47 años. Éstos son datos diferentes de los publicados en diversos estudios ${ }^{14-17}$ en los cuales la mayoría de los pacientes correspondían a hombres. Esta tendencia pudo relacionarse con el influjo humoral sobre la mujer en edad reproductiva secundario al uso de anticonceptivos hormonales o el estado fisiológico del embarazo, similar a lo notificado en estudios mexicanos ${ }^{18,19}$. Destacó la diabetes mellitus, hipertensión arterial y un episodio previo de TVP, de acuerdo con Jacobs, quien encontró que el antecedente personal de TEV es el factor de riesgo más importante relacionado con el paciente $(25 \%)^{20}$. En ese mismo sentido, García, et al. ${ }^{21}$, hallaron como mayor antecedente la TVP en $40 \%$ de sus pacientes. Fue notable la ausencia de factores de riesgo relacionados con TVP, en $25 \%$ de mujeres y $50 \%$ de hombres; esto concuerda con las guías de la Sociedad Europea de Cardiología ${ }^{22}$, en las cuales se menciona que hasta $33 \%$ de los episodios de TVP carece de causa identificable.

La mitad de los pacientes tenía algún antecedente quirúrgico vinculado con TVP y la cirugía ortopédica fue la más frecuente con casi $15 \%$. Asimismo, 20\% de las mujeres del estudio tenían antecedente de cesárea. En cambio, en el estudio PEARL ${ }^{21}$ apenas $12 \%$ de los pacientes tenía algún antecedente quirúrgico reciente, aunque sus registros sobre puerperio o embarazo (4\%) y malignidad $(8 \%)$ fueron similares a los de este estudio. Más del $90 \%$ de los pacientes de esta serie no tenía neoplasia. En contraparte, Jacobs señala que el cáncer y la operación mayor son los principales factores de riesgo para TVP ${ }^{20}$. Al respecto, una revisión de $2018^{21}$ menciona que el TEV contribuye en grado significativo a la morbilidad y mortalidad de los pacientes con cáncer, al multiplicar por tres su posibilidad de sufrir un episodio de TEP letal. Además, las personas con cáncer tienen cinco a siete veces mayor riesgo de TEV y, si su aparición ocurre dentro de los siguientes 12 meses tras el diagnóstico de neoplasia, el pronóstico empeora en grado notable. Sin embargo, Yoon, et al., en un estudio observacional con seis pacientes con cáncer activo en estadio IV, concluyeron que el cáncer no afecta los resultados a corto plazo de la TFM (tratamiento farmacomecánico) en TVP sintomática, aunque la necesidad de stent fuera mayor ${ }^{23}$. 
Tabla 6. Tiempo de seguimiento y descripción de los hallazgos posteriores al egreso de los pacientes

\begin{tabular}{|c|c|c|c|c|c|c|c|}
\hline & & \multicolumn{2}{|c|}{ Masculino } & \multicolumn{2}{|c|}{ Femenino } & \multicolumn{2}{|c|}{ Total } \\
\hline & & $\mathbf{n}$ & $\%$ & $\mathbf{n}$ & $\%$ & $\mathbf{n}$ & $\%$ \\
\hline \multirow[t]{2}{*}{ Controles } & Media & 1.4 & & 1.9 & & 1.7 & \\
\hline & DS & 2.4 & & 1.3 & & 1.7 & \\
\hline \multirow[t]{2}{*}{ Meses de seguimiento } & Media & 3.5 & & 8.2 & & 6.7 & \\
\hline & DS & 4.6 & & 10.2 & & 9.1 & \\
\hline \multirow[t]{6}{*}{ Tiempo de Seguimiento } & $<3$ meses & 0 & 0.0 & 4 & 20 & 4 & 14.3 \\
\hline & 3-6 meses & 2 & 25.0 & 3 & 15.0 & 5 & 17.9 \\
\hline & 6-12 meses & 1 & 12.5 & 4 & 20.0 & 5 & 17.9 \\
\hline & 12-24 meses & 2 & 25.0 & 2 & 10.0 & 4 & 14.3 \\
\hline & Más de 24 meses & 0 & 0.0 & 3 & 15.0 & 3 & 10.7 \\
\hline & No ha tenido seguimiento & 3 & 37.5 & 4 & 20.0 & 7 & 25.0 \\
\hline \multirow[t]{5}{*}{ Calificación de Villalta } & No se aplica & 3 & 42.9 & 4 & 23.5 & 7 & 25.0 \\
\hline & Sin síndrome postrombótico & 4 & 57.1 & 4 & 23.5 & 8 & 28.6 \\
\hline & Síndrome postrombótico leve & 0 & 0.0 & 6 & 35.3 & 6 & 21.4 \\
\hline & Síndrome postrombótico moderado & 0 & 0.0 & 1 & 5.9 & 1 & 3.6 \\
\hline & Síndrome postrombótico grave & 0 & 0.0 & 2 & 11.8 & 2 & 7.1 \\
\hline \multirow[t]{2}{*}{ Muerte } & Sí & 1 & 12.5 & 2 & 10.0 & 3 & 10.7 \\
\hline & No & 7 & 87.5 & 18 & 90.0 & 25 & 89.3 \\
\hline
\end{tabular}

Más del $50 \%$ de los pacientes tenían TVP aguda y fue considerablemente mayor la proporción de mujeres en este grupo; esto delinea una tendencia en los hombres a consultar tardíamente, tal vez porque conceden menor importancia a su autocuidado y a que los síntomas de la TVP, como dolor y edema, pueden confundirse con trastornos recientes, como la operación ortopédica. En el estudio de Lu del 2018, los pacientes con contraindicaciones relativas para trombólisis lograron similares desenlaces con TFM y TDC (trombólisis dirigida por catéter), aunque con una tendencia a mejores resultados en aquellos pacientes con TVP agu$\mathrm{da}^{24}$. El eje venoso más afectado fue el iliofemoral $(>80 \%)$, sea en el diagnóstico por Doppler o por flebografía. Esto puede deberse a la relación con un síndrome venoso compresivo pélvico. Se identificó un nexo significativo entre el compromiso iliofemoral y el antecedente quirúrgico. Estos hallazgos concuerdan con lo notificado en México ${ }^{18}$ y son equiparables a los del estudio PEARL ${ }^{21}$, en el que el segmento venoso más afectado también fue el iliofemoral/poplíteo (31\%). Asimismo, los resultados de estudios de calidad de vida como el de Kahn de 2020 muestran que en pacientes con TVP proximal, la TFM tuvo mejores resultados en seguimientos hasta de 24 meses, respecto de aquellos con compromiso femoropoplíteo ${ }^{25}$.

En una cuarta parte de la población se demostró el síndrome de May-Thurner como factor predisponente para TVP o para dificultar su tratamiento, lo que favorece el desarrollo de SPT, con igual proporción en ambos sexos; se identificó una relación entre el síndrome y la afectación iliofemoral en la flebografía. Según Xue, et al., el síndrome de May-Thurner se considera una de las principales causas de TVP iliofemoral aguda ${ }^{26}$. Algo similar encontró Wang, al publicar una incidencia del síndrome de May-Thurner de $33 \%$ a $67 \%$ en pacientes con TVP27. Sin embargo, esto no siempre es así, ya que en el estudio PEARL ${ }^{21}$ sólo $9 \%$ de la población tenía el síndrome. En esta población se observó una tendencia al uso combinado de trombólisis farmacológica y tromboaspiración, que se empleó en más de $60 \%$ de los hombres y en casi $50 \%$ de las mujeres. Esta propensión se fundamenta en el concepto de Sudheendra ${ }^{28}$, quien sostiene que la TFM acelera la trombólisis y 
disminuye la cantidad de trombolítico usado en comparación con la TDC sola, a través de la generación de un gradiente de presión negativa que da lugar a la aspiración del trombo y que puede administrar el trombolítico a altas presiones y llevar a la fragmentación o aspiración del coágulo. De modo similar, Hager, et al. encontraron esta combinación en $68 \%$ de 79 pacientes ${ }^{29}$; se realizó anglioplastia con o sin stent en más de $75 \%$ de las mujeres y en casi $90 \%$ de los hombres, datos superiores a los de Demartino, et al., que colocaron stent en $60 \%$ de sus pacientes $(n=6)^{14}$. Estos resultados deben compararse de forma cautelosa debido a la amplia diferencia entre el número de pacientes incluidos. Los datos de los autores son superiores también a los del estudio PEARL ${ }^{21}$ y CaVent ${ }^{30}$, con uso de stent en $35 \%$ y $17 \%$, respectivamente.

Poco más del $30 \%$ de la población tuvo complicaciones; es notorio que la mayor parte de ellas correspondió a sangrado menor, en relación con hematomas en los sitios de punción vascular, que no requirieron una intervención adicional. Similares resultados tuvieron $\mathrm{Lu}$, et al., que señalaron que la mayoría de las complicaciones hemorrágicas observadas en el grupo de TDC se conformó con sangrados menores en relación con hematomas en el sitio de punción ${ }^{31}$, y Arriaga que encontró un $33 \%$ de complicaciones ${ }^{18}$. Los autores identificaron complicaciones graves como requerimiento de transfusión de hemocomponentes secundario a descenso de hemoglobina o aparición de hematoma retroperitoneal en casi $10 \%$ de sus pacientes. Tang, et al. en 2019 también reconocieron este fenómeno, pues la intervención endovascular percutánea + anticoagulación incrementó la aparición de episodios de sangrado mayor ${ }^{27}$; y Haig, et al. publicaron tasas similares de complicaciones hemorrágicas graves $(10.6 \%)^{32}$. En un paciente (3.5\%) se presentó un episodio de TEP secundario al procedimiento endovascular, muy inferior al informe de un metaanálisis de 2019, con tasas de TEP posterior al procedimiento de $9.5 \%{ }^{31}$. Una explicación podría ser la elevada frecuencia de uso de TFM y que la mayoría de pacientes cursaba con coágulos de evolución aguda, sin organizar y sin calcificaciones, lo que facilitó su fragmentación y remoción total. En este estudio, 3.5\% de la población tuvo un desenlace letal. Esta cifra se encuentra dentro del promedio expuesto en dos metaanálisis que notificaron una mortalidad de $0.8 \%$ a $7 \%{ }^{15,31}$.

Los pacientes de la serie de los autores se vigilaron en la $\mathrm{UCl}$, donde permanecieron en promedio dos a tres días, en relación con el uso de trombólisis + tromboaspiración, aunque esto podría deberse en parte a que esa fue la técnica endovascular empleada con más frecuencia; y hospitalizaciones que duraron en promedio 15 días. Esto mejora los hallazgos del metaanálisis de Lu, et al. en el que había datos de estancia hospitalaria en 8 de 20 estudios, con un promedio de 46.35 días $^{31}$. Los autores registraron una tasa de retrombosis de casi $30 \%$, que en promedio apareció después de seis meses de realizada la primera intervención. No hubo ninguna recurrencia antes de las 72 $\mathrm{h}$ tras el primer procedimiento. Este resultado se puede deber en gran medida a factores como la mala adherencia a la anticoagulación ambulatoria, sea por desconocimiento, desinterés o falta de acceso al medicamento o los controles médicos, y a la mayor afectación iliofemoral. Esto último, con base en datos de revisiones que sostienen que el compromiso iliofemoral confiere el doble de riesgo de recurrencia, en comparación con la afectación femoropoplítea ${ }^{10}$. En este sentido son superiores los datos de una revisión sistemática de 35 estudios $^{15}, 12$ de ellos con una recurrencia a 30 días de $11.9 \%$. Asimismo, 26 estudios documentaron tasa de retrombosis tardía (> 30 días) de 10.7\% con intervalos de seguimiento de 12 a 24 meses.

La media de seguimiento de los autores fue superior a los ocho meses en las mujeres y de más de tres meses para los hombres, lo cual podría revelar mayor preocupación de parte de las mujeres para continuar su tratamiento. Debe anotarse el escaso tiempo de seguimiento respecto de los informes de un metaanálisis en el que se incluyeron 10 estudios, con un seguimiento promedio de 29.62 meses, aunque dos de los estudios tenían tiempos de seguimiento similares al de los autores o incluso menores (6 meses); en este último se concluyó que la TDC puede mejorar la permeabilidad iliofemoral o SPT grave en comparación con el tratamiento de anticoagulación solo; sin embargo, también aumentó la posibilidad de sangrado, TEP y estancia hospitalaria ${ }^{31}$. La escala de Villalta se aplicó a $75 \%$ de los pacientes, casi 30\% sin SPT y apenas alrededor del $3 \%$ con SPT grave. Esto corresponde con los informes de Tan, et al..$^{33}$ quienes en un metaanálisis de 2019 con seis estudios y 350 pacientes encontraron que la TFM redujo la calificación de Villalta, en comparación con la TDC sola. Los resultados de los autores son inferiores a lo notificado en un estudio mexicano que comparó el uso de TDC con anticoagulación para reducir el SPT y en el que cerca del $60 \%$ de los pacientes a dos años no presentó SPT ${ }^{19}$. Asimismo, Hager, et al. ${ }^{28}$ propusieron la TFM para TVP proximal como tratamiento único o combinado con TDC para prevenir el SPT. El estudio $\mathrm{CaVent}^{29}$ demostró que la 
TDC mejora los resultados clínicos a largo plazo al reducir el SPT, en comparación con la anticoagulación convencional y las medias de compresión elástica, aunque este efecto se obtuvo a costa de un pequeño aumento del riesgo de sangrado.

Este estudio posee limitaciones, como una muestra pequeña, tiempo de seguimiento corto e imposibilidad para aplicar la calificación de Villalta a toda la población. Dentro de las fortalezas debe señalarse un claro conocimiento y aplicación de las recomendaciones internacionales ${ }^{10,11,34}$ y nacionales ${ }^{9}$ acerca del tratamiento intervencionista para TVP, así como ser el estudio con la población más grande de pacientes en el plano nacional y el primero en el suroccidente del país.

\section{Conclusión}

Este estudio sugiere que el uso de TFM + TDC ayuda al tratamiento de la TVP, al reducir los tiempos de estancia hospitalaria, la ocupación de UCl y la aparición de SPT grave, con igual riesgo de complicaciones hemorrágicas mayores y tasa de mortalidad, y mayor riesgo de retrombosis, en comparación con las publicaciones médicas mundiales actuales.

\section{Agradecimientos}

Los autores agradecen a la Clínica La Estancia, Popayán, Cauca, Departamento de Cirugía, Universidad del Cauca; al Dr. Jorge Augusto Herrera (MD, Esp. Cirugía General, Director científico, Clínica La Estancia, Popayán, Cauca); a la Dra. Nazly Ipia (MD, Servicio de Angiografía, Clínica La Estancia, Popayán, Cauca), Soluciones vasculares, Popayán, Cauca.

\section{Conflicto de intereses}

Ninguno.

\section{Financiamiento}

Ninguno.

\section{Responsabilidades éticas}

Protección de personas y animales. Los autores declaran que los procedimientos seguidos se adecuaron a las normas éticas del comité de experimentación humana responsable y a la Asociación Médica Mundial y la Declaración de Helsinki.
Confidencialidad de los datos. Los autores declaran que han seguido los protocolos de su centro de trabajo sobre la publicación de datos de pacientes.

Derecho a la privacidad y consentimiento informado. Los autores han obtenido el consentimiento informado de los pacientes o sujetos referidos en el artículo. Este documento obra en poder del autor de correspondencia.

\section{Bibliografía}

1. Patel K, Fasanya A, Yadam S, Dumont T. Pathogenesis and epidemiology of venous thromboembolic disease. Crit Care Nurs Q. 2017;40(3):191200.

2. Lorenzo-López L, Núñez-Naveira L, Millán-Calenti JC. Enfermedad tromboembólica venosa en personas mayores: revisión de la literatura. Venous thromboembolism in elderly people: GEROKOMOS. 2014;25(3):93-7.

3. Dennis R. Estudio nacional sobre tromboembolismo venoso en población hospitalaria en Colombia. Acta Medica Colomb [Internet]. 1996;21:55-63. Available from: http://actamedicacolombiana.com/anexo/articulos/02-1996-02-Estudio_nacional_sobre_tromboembolismo_venoso_ en.pdf

4. Porres-Aguilar M, Burgos JD, Muñoz OC, Soto-Cora E, Mukherjee D. Successful pharmacomechanical intervention with ultrasonic-accelerated thrombolytic catheter for massive pulmonary embolism. Indian Heart $J$ [Internet]. 2013;65(6):699-702. Available from: http://dx.doi.org/10.1016/j. ihj.2013.10.007

5. Kahn SR, Comerota AJ, Cushman M, Evans NS, Ginsberg JS, Goldenberg NA, et al. The postthrombotic syndrome: evidence-based prevention, diagnosis, and treatment strategies: ascientific statement from the American Heart Association. Circulation. 2014;130(18):1636-61.

6. Robertson L, Mcbride O, Burdess A. Pharmacomechanical thrombectomy for iliofemoral deep vein thrombosis (Review). Cochrane Libr. 2016.

7. Bell EJ, Lutsey PL, Basu S, Cushman M, Heckbert SR, Lloyd-Jones DM, et al. Lifetime risk of venous thromboembolism in two cohort studies. Am J Med. 2016;129(3):339.e19-26. doi: 10.1016/j.amjmed.2015.10.014.

8. Jenkins JS. Endovascular therapies to treat iliofemoral deep venous thrombosis. Prog Cardiovasc Dis. 2011;54(1):70-6. doi: 10.1016/j. pcad.2011.03.008

9. Hernández C, Mauricio J, Mu JF, Cadavid G. Consenso colombiano de fibrinólisis selectiva con catéter en enfermedad vascular tromboembólica. Rev Colomb Cardiol. 2020;27(1).

10. Meissner $\mathrm{MH}$, Gloviczki $\mathrm{P}$, Comerota AJ, Dalsing MC, Eklof BG, Gillespie DL, et al. Early thrombus removal strategies for acute deep venous thrombosis: Clinical Practice Guidelines of the Society for Vascular Surgery and the American Venous Forum. J Vasc Surg [Internet]. 2012;55(5):1449-62. Available from: http://dx.doi.org/10.1016/j. jvs.2011.12.081

11. Kearon C, Akl EA, Ornelas J, Blaivas A, Jiménez D, Bounameaux $\mathrm{H}$, et al Antithrombotic therapy for VTE disease: CHEST guideline and expert panel report. Chest. 2016;149(2):315-52.

12. Kearon SS. Definition of major bleeding in clinical investigations of antihemostatic medicinal products in non-surgical patients. J Thromb Haemost. 2005;692-4.

13. Taggart $D$, Valgimigli M, Bhatt DL, Ohman EM, Menon V, Rao S V, et al. Standardized bleeding definitions for cardiovascular clinical trials. Circulation. 2011;123(23):2736-47

14. López R, DeMartino R, Fleming M, Bjarnason H, Neisen M. Aspiration thrombectomy for acute iliofemoral or central deep venous thrombosis. J Vasc Surg Venous Lymphat Disord [Internet]. 2019;7(2):162-8. Available from: https://doi.org/10.1016/j.jvsv.2018.09.015

15. Wang W, Sun R, Chen Y, Liu C. Meta-analysis and systematic review of percutaneous mechanical thrombectomy for lower extremity deep vein thrombosis. J Vasc Surg [Internet]. 6(6):788-800. Available from: https:// doi.org/10.1016/j.jvsv.2018.08.002

16. Vedantham S, Goldhaber SZ, Julian JA, Kahn SR, Jaff MR, Cohen DJ, et al. Pharmacomechanical catheter-directed thrombolysis for deep-vein thrombosis. N Engl J Med. 2017;377(23):2240-52.

17. Sharifi M, Bay C, Mehdipour M, Sharifi J, TORPEDO I. Thrombus Obliteration by Rapid Percutaneous Endovenous Intervention in Deep Venous Occlusion (TORPEDO) trial: midterm results. J Endovasc Ther [Internet]. 2012;19(2):273-80. Available from: http://sfx.scholarsportal. info $/ \mathrm{mcmaste}$ ? sid=OVID: medline \&id $=$ pmid: $22545895 \& i d=-$ doi:10.1583/11-3674MR.1\&issn = 1526-6028\&isbn = \&volume=19\&issue $=2 \&$ spage $=273 \&$ pages $=273-80 \&$ date $=2012 \&$ title $=$ Journal + of + En dovascular+Therapy\&atitle=Thrombus+Obliteration+by+Rapid+Percut 
18. Arriaga-Caballero JE, Guardado-Bermúdez F, Flores-Escartín MH, Sánchez-Nicolat NE, Serrano-Lozano JA, Álvarez-Arcaute NR, et al. Complicaciones asociadas a uso de filtro de vena cava en pacientes con trombosis venosa profunda. Rev Mex Angiol. 2019;47(2):64-9.

19. Carrasco-Alamilla RA, Flores-Ramírez C, López-Ríos MR Reyes-Chávez SE. Trombólisis dirigida por catéter para la prevención del síndrome postrombótico en trombosis venosa profunda del segmento iliocavo (catheter-directed thrombolysis of the iliocaval segment in the prevention of the post-thrombotic syndrome). Rev Mex Angiol. 2019;47 (3):16-21.

20. Jacobs B, Henke P. Evidence-based therapies for pharmacologic prevention and treatment of acute deep vein thrombosis and pulmonary embolism. Surg Clin N Am. 2018;98:239-53.

21. García MJ, Lookstein R, Malhotra R, Amin A, Blitz LR, Leung DA, et al. Endovascular management of deep vein thrombosis with rheolytic thrombectomy: final report of the Prospective Multicenter PEARL Registry. $J$ Vasc Interv Radiol [Internet]. 2015;1-9. Available from: http://dx.doi.org/10.1016/j.jvir.2015.01.036

22. Konstantinides S, Torbicki A, Agnelli G, Danchin N, Fitzmaurice D, Galie N, et al. 2014 ESC Guidelines on the diagnosis and management of acute pulmonary embolism-web addenda The Task Force for the Diagnosis and Management of Acute Pulmonary Embolism of the European Society of Cardiology (ESC). Eur Heart J. 2014.

23. Yoon WJ, Halandras P, Aulivola B, Crisostomo P. Malignancy does not affect outcomes of pharmacomechanical thrombolysis in acute symptomatic iliofemoral deep vein thrombosis. Ann Vasc Surg [Internet] 2018;51:234-8. Available from: https://doi.org/10.1016/j.avsg. 2018.01.081

24. Liu X, Cao P, Li Y, Zhao J, Li L, Li H, et al. Safety and efficacy of pharmacomechanical thrombolysis for acute and subacute deep vein thrombosis patients with relative contraindications. Medicine (Baltimore) 2018;0:0-5.

25. Kahn SR, Julian JA, Kearon C, Gu CS, Cohen DJ, Magnuson EA, et al Quality of life after pharmacomechanical catheter-directed thrombolysis for proximal deep venous thrombosis. J Vasc Surg Venous Lymphat Disord. 2020;8(1):8-23.e18.
26. Xue G, Huang X, Ye M, Liang W, Zhang H, Zhang J. Catheter-directed thrombolysis and stenting in the treatment of iliac vein compression syndrome with acute iliofemoral deep vein thrombosis : outcome and follow-up. Ann Vasc Surg [Internet]. 2014;28(4):957-63. Available from: http://dx.doi.org/10.1016/j.avsg.2013.11.012

27. Wang C, Deng H. Percutaneous endovenous intervention plus anticoagulation versus anticoagulation alone for treating patients with proximal deep vein thrombosis: a meta-analysis and systematic review. Ann Vasc Surg [Internet]. 2018; Available from: https://doi.org/10.1016/j.avsg.2017.09.027

28. Sudheendra D, Vedantham S. Catheter directed therapy options for iliofemoral venous thrombosis. Surg Clin N Am. 2018;98:255-65

29. Hager E, Yuo T, Avgerinos E, Naddaf A, Jeyabalan G, Marone L, et al. Anatomic and functional outcomes of pharmacomechanical and catheter-directed thrombolysis of iliofemoral deep venous thrombosis. $\mathrm{J}$ Vasc Surg Venous Lymphat Disord [Internet]. 2014;2(3):246-52. Available from: http://dx.doi.org/10.1016/j.jvsv.2014.02.003

30. Enden T, Haig Y, Kløw N, Slagsvold C, Sandvik L, Ghanima W, et al. Long-term outcome after additional catheter-directed thrombolysis versus standard treatment for acute iliofemoral deep vein thrombosis (the CaVenT study ): a randomised controlled trial. Lancet. 379(9810):31-8.

31. Lu Y, Chen L, Chen J, Tang T. Catheter-directed thrombolysis versus standard anticoagulation for acute lower extremity deep vein thrombosis: a meta-analysis of clinical trials. 2018;(1).

32. Haig Y, Enden T, Slagsvold CE, Sandvik L, Sandset PM, Kløw NE. Determinants of early and long-term efficacy of catheter-directed thrombolysis in proximal deep vein thrombosis. J Vasc Interv Radiol [Internet]. 2013;24(1):1724. Available from: http://dx.doi.org/10.1016/j.jvir.2012.09.023

33. Tang T, Chen L, Chen J, Mei T, Lu Y. Pharmacomechanical thrombectomy versus catheter-directed thrombolysis for iliofemoral deep vein thrombosis: a meta-analysis of clinical trials. Clin Appl Thromb. 2019.

34. Vedantham S, Millward SF, Cardella JF, Hofmann LV, Razavi MK, Grassi CJ, et al. Society of Interventional Radiology Position Statement: treatment of acute iliofemoral deep vein thrombosis with use of adjunctive catheter-directed intrathrombus thrombolysis. J Vasc Interv Radiol [Internet]. 2009;20(7 SUPPL.):S332-5. Available from: http://dx.doi.org/10.1016/j.jvir.2009.04.017 